OPEN ACCESS

Edited by:

Robert Ohgami,

University of California, San Francisco,

United States

Reviewed by:

Christian M. Capitini,

University of Wisconsin-Madison,

United States

Harinder Gill,

University of Hong Kong, Hong Kong

${ }^{*}$ Correspondence: Wenbin Qian

gianwenb@zju.edu.cn

Xuzhao Zhang

zxzzju@zju.edu.cn

Specialty section: This article was submitted to Hematologic Malignancies,

a section of the journal

Frontiers in Oncology

Received: 11 April 2021 Accepted: 28 May 2021

Published: 05 July 2021

Citation:

Zhang X, Chen J, Wang W, LiX, Tan Y, Zhang X and Qian W (2021) Treatment

of Central Nervous System Relapse in Acute Promyelocytic Leukemia by Venetoclax: A Case Report.

Front. Oncol. 11:693670. doi: 10.3389/fonc.2021.693670

\section{Treatment of Central Nervous System Relapse in Acute Promyelocytic Leukemia by Venetoclax: A Case Report}

\author{
Xuzhao Zhang ${ }^{1 *}$, Jinliang Chen ${ }^{2}$, Weiqin Wang ${ }^{1}$, Xian Li $^{1}$, Yanbin Tan $^{3}$, Xiaohong Zhang ${ }^{1}$ \\ and Wenbin Qian ${ }^{1 *}$ \\ ${ }^{1}$ Department of Hematology, The Second Affiliated Hospital of Zhejiang University School of Medicine, Hangzhou, China, \\ ${ }^{2}$ Center of Clinical Pharmacology, The Second Affiliated Hospital of Zhejiang University School of Medicine, Hangzhou, China, \\ ${ }^{3}$ Department of Radiology, The Second Affiliated Hospital of Zheiliang University School of Medicine, Hangzhou, China
}

Extramedullary relapse of acute promyelocytic leukemia is a rare phenomenon and is associated with a poor prognosis, with the central nervous system being the most common site of relapse. The current treatments are still limited. Venetoclax, a selective inhibitor of BCL2, is a small molecule that can cross the blood-brain barrier and shows a potential efficacy in the treatment of chronic lymphocytic leukemia with central nervous system involvement. Although venetoclax has also been used in the treatment of acute myeloid leukemia in recent years, there are no reports of its use in the treatment of central nervous system relapse in acute promyelocytic leukemia. Here, we report a case of central nervous system relapse in acute promyelocytic leukemia that achieved complete remission after oral treatment with venetoclax. The presence of venetoclax in the patient's CSF was confirmed by testing CSF and plasma by mass spectrometry. The concentration of venetoclax in CSF was approximately 1/300 of that in plasma trough concentration. The treatment experience in this case demonstrates the potential ability of venetoclax to treat of central nervous system relapse/involvement in acute promyelocytic leukemia, thus providing a new treatment option for this kind of patient.

Keywords: acute promyelocytic leukemia, central nervous system, relapse, venetoclax, CSF concentration

\section{INTRODUCTION}

Since the use of all-trans retinoic acid (ATRA) and arsenic trioxide (ATO) in the treatment of acute promyelocytic leukemia (APL), there have been extraordinary advances in the efficacy of APL treatment (1-4). Extramedullary relapse of APL is a rare phenomenon and is associated with a poor prognosis, with the central nervous system (CNS) being the most common site of relapse $(5,6)$. Median survival in patients with CNS relapse is shorter than that in patients with extracentral recurrences alone (5). Current treatments, including intrathecal chemotherapy, systemic chemotherapy with high-dose methotrexate (MTX) and/or cytarabine, radiation therapy and ATO (7), for CNS relapse in APL are still limited. Venetoclax, a selective inhibitor of BCL2, is a small molecule that can cross the blood-brain barrier and shows potential efficacy in the treatment 
of chronic lymphocytic leukemia (CLL) with CNS involvement (8). Although venetoclax has also been used in the treatment of acute myeloid leukemia (AML) in recent years $(9,10)$, there are no reports of its use in the treatment of CNS relapse in APL. Here, we report a case of CNS relapse in APL that achieved successful clearance of cerebrospinal fluid (CSF) APL cells after oral treatment with venetoclax. We hope to provide a new approach to the treatment of patients with CNS relapse in APL.

\section{CASE PRESENTATION}

A 63-year-old male patient was admitted to our hospital with "diagnosed APL for more than 6 years and visual double vision for more than 2 months." The patient had no family history and no specific social history. More than 6 years ago, this patient went to the local hospital for "recurrent gingival bleeding" and was diagnosed with APL in the low-risk group after providing the relevant examination. The patient received ARTA+ATO initial induction therapy, stopped ATO because of liver function impairment and switched to idarubicin and cytarabine therapy, which resulted in complete remission. Subsequently, the patient received intermittent consolidation and maintenance therapy with retinoic acid-arsenic acid. However, over the past 6 years, the patient had 4 relapses, obtaining remission after each relapse with salvage chemotherapy (details unknown). More than 2 months ago (APL in remission status), the patient had transient loss of consciousness without obvious inducement, which remitted spontaneously after a few moments. When he went to the local hospital, no typical epileptiform discharge was seen on electroencephalogram (EEG) and no obvious abnormality was seen on cranial magnetic resonance imaging (MRI). During the hospitalization, there was double vision, and a bone marrow smear revealed $1.57 \%$ promyelocytes. PML/RAR $\alpha$ fusion gene test was negative. The lumbar puncture CSF test was not significantly abnormal. Cotton wool spot found on fundus examination was considered to be related to APL. During hospitalization, the patient had several epileptic-like seizures, and the symptoms improved slightly after neurology consultation with sodium valproate and mannitol to lower the cranial pressure. The patient was transferred to our hospital and was admitted to our department for further treatment.

Laboratory tests: Complete blood count: white blood cell count 3 $600 / \mu \mathrm{L}$, hemoglobin $134 \mathrm{~g} / \mathrm{L}$, and platelet count $191000 / \mu \mathrm{L}$. The coagulation profile, serum biochemical tests, and tumor markers (including AFP, CEA, CA199, CA125, CA242, NSE, CA211, SCC and PSA) were all unremarkable. Bone marrow smear: no obvious leukemic cells were seen; the PML-RAR $\alpha$ fusion gene was positive in bone marrow sample (215.49 copies, PML-RAR $\alpha /$ ABL 0.001); bone marrow immunophenotyping: no obvious abnormal immune-phenotypes were seen. Peripheral blood was negative for the PML-RAR $\alpha$ fusion gene. This patient subsequently

Abbreviations: APL, acute promyelocytic leukemia; CNS, central nervous system; AML, acute myeloid leukemia; ATRA, all-trans retinoic acid; ATO, arsenic trioxide; MTX, methotrexate; CLL, chronic lymphocytic leukemia; CSF, cerebrospinal fluid; EEG, electroencephalogram; MRI, magnetic resonance imaging; HSCT, hematopoietic stem cell transplantation. underwent a lumbar puncture on July 16, 2019 with a CSF pressure of $300 \mathrm{mmH} 2 \mathrm{O}, 150$ nucleated cells/ $\mu \mathrm{L}$ (normal reference value $<8 / \mu \mathrm{L}$ ), $15 \%$ neutrophils, $60 \%$ lymph, and $25 \%$ mononuclear. CSF exfoliation cytology found abnormal promyelocytic leukemia cells (Figures 1-A1, 2). The PML-RAR $\alpha$ fusion gene was positive in CSF (647 000 copies, PML-RAR $\alpha / A B L ~ 1.174)$. CSF flow cytometry testing: Abnormal promyelocytic leukemia cells account for approximately $77.475 \%$ of leukocytes. Diagnosis: 1 . CNS leukemia (secondary to APL); 2. APL.

The patient was treated with methotrexate, cytarabine and dexamethasone twice weekly intrathecal injections in combination with ATO and mannitol continuous intravenous alternating therapy for 30 days and the PML-RAR $\alpha$ fusion gene was negative both in the CSF and bone marrow. The patient refused autologous hematopoietic stem cell transplantation (HSCT) consolidation for personal reasons. Subsequently, the patient received intermittent high-dose methotrexate and cytarabine combination chemotherapy and intrathecal injections of methotrexate, cytarabine and dexamethasone. Unfortunately, the patient has had a recurrence of head swelling since May 2020, and repeat lumbar puncture and cranial enhancement MRI suggested another intracranial relapse. Failure to induce remission again in this patient by triple intrathecal injection of methotrexate, cytarabine and dexamethasone in combination with ATO and mannitol continuous intravenous therapy. High-dose methotrexate in combination with medium-dose cytarabine also failed to work and resulted in severe myelosuppression. Then, the patient tried venetoclax oral treatment initially at a dosage of $100 \mathrm{mg}$ once a day, and gradually increased to $400 \mathrm{mg}$ once a day. After taking venetoclax for two weeks while continuing triple IT therapy, the patient's head swelling was miraculously relieved, and a repeat lumbar puncture four weeks later showed complete disappearance of CSF leukemia cells and immunological remission of the bone marrow was also maintained (Figure 2). CSF leukemic cells continued to be negative by flow cytometry, but a repeat MRI of the brain still suggested possible localized meningeal involvement (Figures 1-B1-3). The presence of venetoclax in the patient's CSF was confirmed by testing CSF and plasma specimens when the patient was on venetoclax for 4 weeks. The concentration of venetoclax in CSF was approximately $1 / 300$ of that in the plasma trough concentration (Figure 3). This patient is still on ongoing venetoclax therapy and has maintained complete remission of CNS for 8 months (Figure 2). He is still undergoing regular follow-up. To the best of our knowledge, this is the first case of venetoclax applied to CNS relapse of APL.

\section{DISCUSSION}

CNS relapse is a low incidence event in APL but is a factor significantly associated with poor prognosis. Similar to the present case, CNS relapse in APL can occur several years after achieving CR and is often difficult to manage. Although there are very few cohort studies to try new treatment strategies for CNS relapse in APL, current experience in the treatment of this situation is overwhelmingly based on case reports. Therefore, 


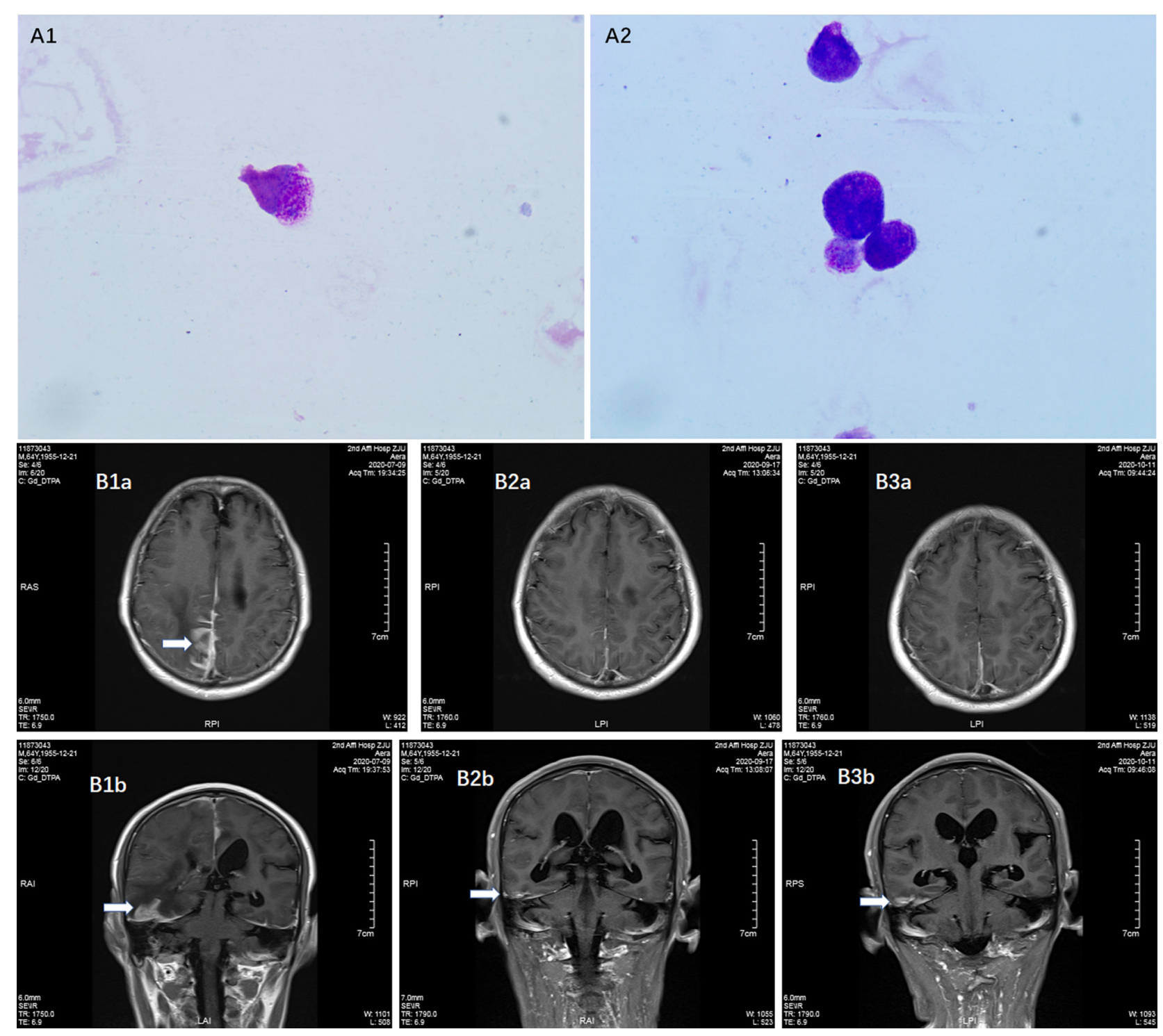

FIGURE 1 | Detection of meningeal involvement by APL cells. (A1-2) Promyelocytic leukemia cells in CSF; (B1a, 1b) Contrast MRI before venetoclax treatment on July 9, 2020, (B2a, 2b) Contrast MRI during venetoclax treatment on September 17, 2020; (B3a, 3b) Contrast MRI during venetoclax treatment on November 11, 2020.

finding new therapeutic measures is an urgent challenge that needs to be addressed.

The Bcl-2 inhibitor venetoclax is a small molecule that has shown promising therapeutic effects in $\operatorname{AML}(9,10)$ and lymphatic malignancies (11-13). There are also few case reports of the use of venetoclax in cases of CNS involvement in CLL (8). In vitro experiments revealed that the APL cell line NB4 has good sensitivity to venetoclax (14). However, to date there have been no reports of venetoclax for the treatment of CNS involvement/ relapse in APL.

This is a refractory case of APL with CNS relapse, which did not respond well to intrathecal injection and high-dose MTX-based regimens. In this case, venetoclax showed excellent anti-meningeal APL efficacy and was well tolerated with ongoing triple IT therapy. A recent study found that venetoclax can cross the blood-brain barrier and exert antimembranous CLL activity, and its CSF concentration is approximately $1 / 1000$ of the plasma concentration (8). In this study, we also demonstrated that the presence of venetoclax in CSF can be detected by mass spectrometry, and the concentration in CSF is approximately 1/ 300 of the plasma trough concentration. This finding could explain the effectiveness of venetoclax for meningeal APL. However, the lack of controls limits the absolute quantification of venetoclax concentrations in plasma and CSF.

Although this patient's brain MRI still suggested possible localized meningeal involvement, the experience of this case offers a new option for the treatment strategy of CNS involvement/relapse in APL as well as other kinds of AML. 


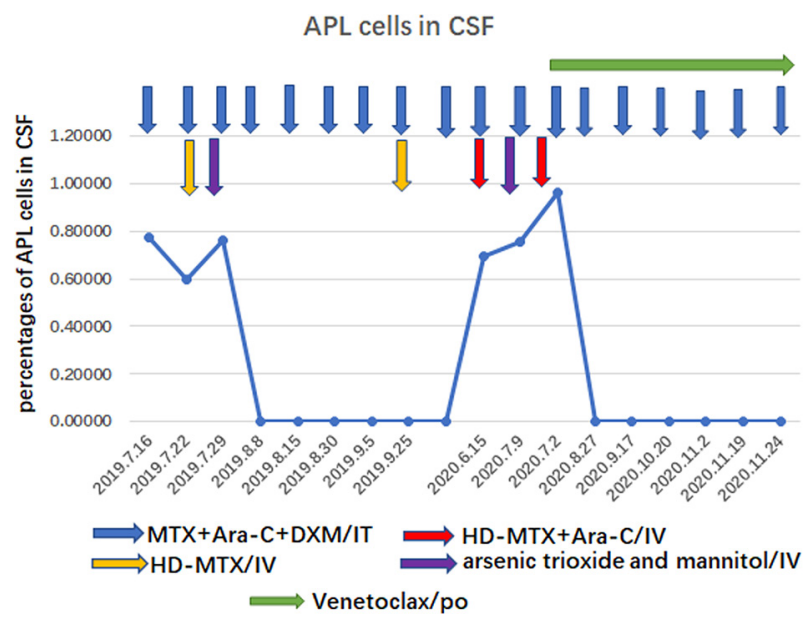

FIGURE 2 | Trends of APL cells counts in CSF.

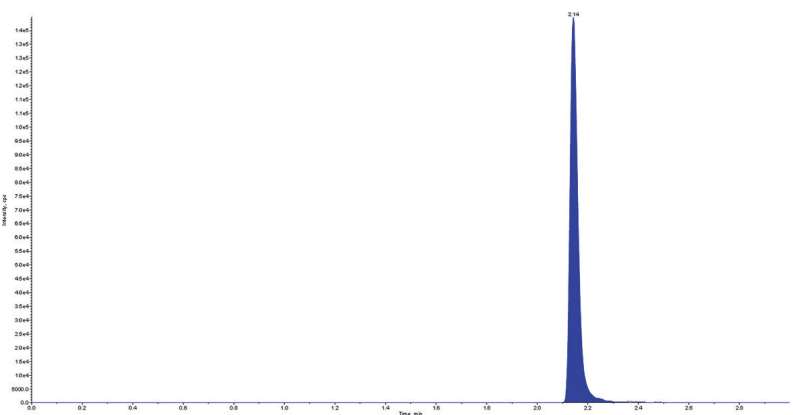

B

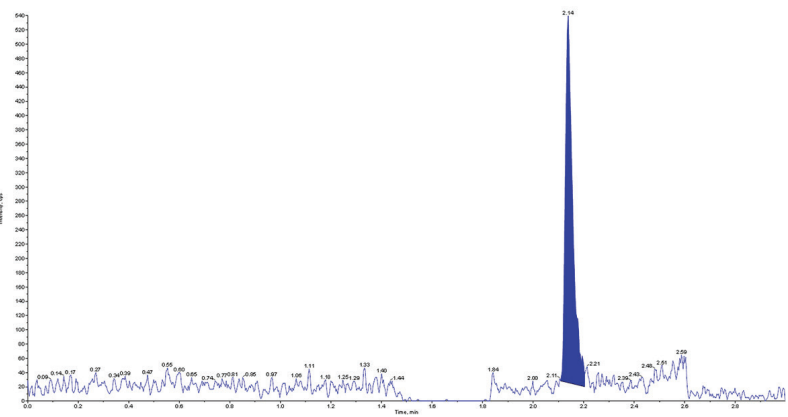

C

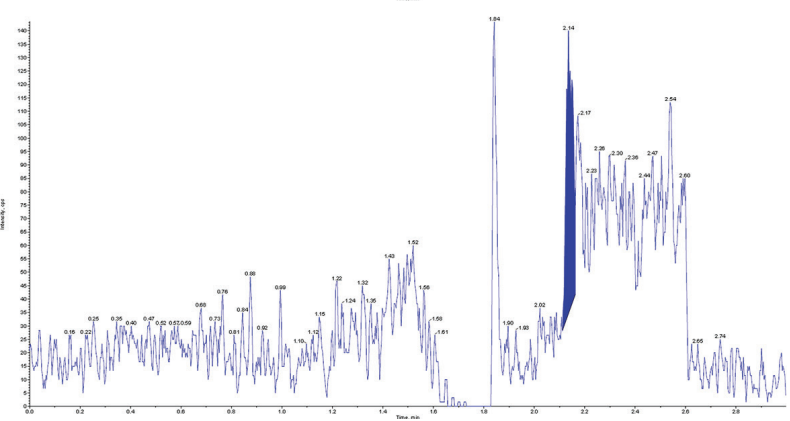

FIGURE 3 | Detection of venetoclax in plasma and CSF by mass spectrometry. (A) Plasma venetoclax valley concentration; (B) Venetoclax concentration in the patient's CSF; (C) Background concentration of venetoclax in the patient's CSF before starting venetoclax. 
However, the treatment experience in this case also suggests that single agent venetoclax therapy still seems to be inadequate and more effective combination therapy options still need to be explored to improve treatment efficacy. This case is only an empirical report of a single case, and the efficacy of venetoclax in CNS APL involvement will need to be confirmed in a large clinical study.

\section{DATA AVAILABILITY STATEMENT}

The original contributions presented in the study are included in the article/supplementary material. Further inquiries can be directed to the corresponding authors.

\section{ETHICS STATEMENT}

The studies involving human participants were reviewed and approved by the ethics committee of the Second Affiliated Hospital of Zhejiang University School of Medicine. The patients/participants provided their written informed consent to participate in this study. Written informed consent was

\section{REFERENCES}

1. Shen ZX, Shi ZZ, Fang J, Gu BW, Li JM, Zhu YM, et al. All-Trans Retinoic Acid/As2O3 Combination Yields a High Quality Re-Mission and Survival in Newly Diagnosed Acute Promyelocytic Leukemia. Proc Natl Acad Sci USA (2004) 101:5328-35. doi: 10.1073/pnas.0400053101

2. Hu J, Liu YF, Wu CF, Xu F, Shen ZX, Zhu YM, et al. Long-Term Efficacy and Safety of All-Trans Retinoic Acid/Arsenic Trioxide-Based Therapy in Newly Diagnosed Acute Promyelocytic Leukemia. Proc Natl Acad Sci USA (2009) 106:3342-7. doi: 10.1073/pnas.0813280106

3. Zhu H, Hu J, Li X, Chen L, Zhao H, Zhou W, et al. All-Trans Retinoic Acid and Arsenic Combination Therapy Benefits Low-to-Intermediate-Risk Patients With Newly Diagnosed Acute Promyelocytic Leukaemia: A LongTerm Follow-Up Based on Multivariate Analysis. Br J Haematol (2015) 171:277-80. doi: 10.1111/bjh.13375

4. Zhu H, Hu J, Chen L, Zhou W, Li X, Wang L, et al. The 12-Year Follow-Up of Survival, Chronic Adverse Effects, and Retention of Arsenic in Patients With Acute Promyelocytic Leukemia. Blood (2016) 128:1525-8. doi: 10.1182/ blood-2016-02-699439

5. de Botton S, Sanz MA, Chevret S, Dombret H, Martin G, Thomas X, et al. Extramedullary Relapse in Acute Promyelocytic Leukemia Treated With AllTrans Retinoic Acid and Chemotherapy. Leukemia (2006) 20:35-41. doi: 10.1038/sj.leu.2404006

6. Albano F, Specchia G. Extramedullary Disease in Acute Promyelocytic Leukemia: Two-in-One Disease. Mediterr J Hematol Infect Dis (2011) 3: e2011066. doi: 10.4084/mjhid.2011.066

7. Wang H, Cao F, Li J, Li L, Li Y, Shi C, et al. Arsenic Trioxide And Mannitol for The Treatment of Acute Promyelocytic Leukemia Relapse in the Central Nervous System. Blood (2014) 124(12):1998-2000. doi: 10.1182/ blood-2014-04-568121

8. Reda G, Cassin R, Dovrtelova G, Matteo C, Giannotta J, D’Incalci M, et al. Venetoclax Penetrates in Cerebrospinal Fluid and May Be Effective in Chronic Lymphocytic Leukemia With Central Nervous System Involvement. Haematologica (2019) 104(5):e222-3. doi: 10.3324/haematol.2018.213157

9. DiNardo CD, Pratz KW, Letai A, Jonas BA, Wei AH, Thirman M, et al. Safety and Preliminary Efficacy of Venetoclax With Decitabine or Azacitidine in obtained from the individual(s) for the publication of any potentially identifiable images or data included in this article.

\section{AUTHOR CONTRIBUTIONS}

XuZ and WQ designed the study. XuZ, WW, XL, and XiZ collected the data. WW performed the cytology analysis of APL cells in CSF. JC performed the analysis of venetoclax in plasma and CSF. YT performed the MRI analysis. XuZ and WQ wrote the manuscript. All authors contributed to the article and approved the submitted version.

\section{FUNDING}

This work was supported by a grant from the National Natural Science Foundation of China (81000895 to XuZ) and a grant from Zhejiang Provincial Natural Science Foundation of China (LY14H160032 to XuZ).

\section{ACKNOWLEDGMENTS}

We thank other involved research technicians, data managers and physicians for their contribution to this manuscript.

Elderly Patients With Previously Untreated Acute Myeloid Leukaemia: A Non-Randomised, Open-Label, Phase 1b Study. Lancet Oncol (2018) 19 (2):216-28. doi: 10.1016/S1470-2045(18)30010-X

10. DiNardo CD, Jonas BA, Pullarkat V, Thirman MJ, Garcia JS, Wei AH, et al. Azacitidine and Venetoclax in Previously Untreated Acute Myeloid Leukemia. N Engl J Med (2020) 383(7):617-29. doi: 10.1056/ NEJMoa2012971

11. Roberts AW, Davids MS, Pagel JM, Kahl BS, Puvvada SD, Gerecitano JF, et al. Targeting BCL2 With Venetoclax in Relapsed Chronic Lymphocytic Leukemia. N Engl J Med (2016) 374(4):311-22. doi: 10.1056/ NEJMoa1513257

12. Davids MS, Roberts AW, Seymour JF, Pagel JM, Kahl BS, Wierda WG, et al. Phase I First-in-Human Study Of Venetoclax in Patients With Relapsed or Refractory non-Hodgkin Lymphoma. J Clin Oncol (2017) 35(8):826-33. doi: 10.1200/JCO.2016.70.4320

13. Eyre TA, Walter HS, Iyengar S, Follows G, Cross M, Fox CP, et al. Efficacy Of Venetoclax Monotherapy in Patients With Relapsed, Refractory Mantle Cell Lymphoma After Bruton Tyrosine Kinase Inhibitor Therapy. Haematologica (2019) 104(2):e68-71. doi: 10.3324/haematol. 2018.198812

14. Niu X, Wang G, Wang Y, Caldwell JT, Edwards H, Xie C, et al. Acute Myeloid Leukemia Cells Harboring MLL Fusion Genes or With the Acute Promyelocytic Leukemia Phenotype are Sensitive to the Bcl-2-selective Inhibitor ABT-199. Leukemia (2014) 28(7):1557-60. doi: 10.1038/leu.2014.72

Conflict of Interest: The authors declare that the research was conducted in the absence of any commercial or financial relationships that could be construed as a potential conflict of interest.

Copyright (c) 2021 Zhang, Chen, Wang, Li, Tan, Zhang and Qian. This is an openaccess article distributed under the terms of the Creative Commons Attribution License (CC BY). The use, distribution or reproduction in other forums is permitted, provided the original author(s) and the copyright owner(s) are credited and that the original publication in this journal is cited, in accordance with accepted academic practice. No use, distribution or reproduction is permitted which does not comply with these terms. 